\title{
Search-related suppression of hippocampus and default network activity during associative memory retrieval
}

\author{
Emilie T. Reas' ${ }^{1}$, Sarah I. Gimbel ${ }^{1}$, Jena B. Hales ${ }^{1}$ and James B. Brewer ${ }^{1,2}$ * \\ ${ }^{1}$ Department of Neurosciences, University of California, San Diego, CA, USA \\ ${ }^{2}$ Department of Radiology, University of California, San Diego, CA, USA
}

\section{Edited by:}

Neal J. Cohen, University of Illinois, USA

\section{Reviewed by:}

Marian Berryhill, University of Nevada Reno, USA

R. Alison Adcock, Duke University, USA

*Correspondence:

James B. Brewer, Human Memory Laboratory, 8950 Villa La Jolla Drive C212, La Jolla, CA 92037, USA. e-mail: jbrewer@ucsd.edu

\begin{abstract}
Episodic memory retrieval involves the coordinated interaction of several cognitive processing stages such as mental search, access to a memory store, associative re-encoding, and post-retrieval monitoring. The neural response during memory retrieval is an integration of signals from multiple regions that may subserve supportive cognitive control, attention, sensory association, encoding, or working memory functions. It is particularly challenging to dissociate contributions of these distinct components to brain responses in regions such as the hippocampus, which lies at the interface between overlapping memory encoding and retrieval, and "default" networks. In the present study, event-related functional magnetic resonance imaging (fMRI) and measures of memory performance were used to differentiate brain responses to memory search from subcomponents of episodic memory retrieval associated with successful recall. During the attempted retrieval of both poorly and strongly remembered word pair associates, the hemodynamic response was negatively deflected below baseline in anterior hippocampus and regions of the default network. Activations in anterior hippocampus were functionally distinct from those in posterior hippocampus and negatively correlated with response times. Thus, relative to the pre-stimulus period, the hippocampus shows reduced activity during intensive engagement in episodic memory search. Such deactivation was most salient during trials that engaged only pre-retrieval search processes in the absence of successful recollection or post-retrieval processing. Implications for interpretation of hippocampal fMRI responses during retrieval are discussed. A model is presented to interpret such activations as representing modulation of encoding-related activity, rather than retrieval-related activity. Engagement in intensive mental search may reduce neural and attentional resources that are otherwise tonically devoted to encoding an individual's stream of experience into episodic memory.
\end{abstract}

Keywords: fMRI, hippocampus, default network, memory, retrieval

\section{INTRODUCTION}

Functional imaging has the potential to dissect the influences of integrated, but separable, neural processes contributing to successful recall; however, the challenge remains to identify and isolate such components and then dissociate these rapid, transient processes. Human and animal studies implicate the hippocampus in the encoding and retrieval of episodic memories (Lepage et al., 1998; Tulving and Markowitsch, 1998; Eichenbaum, 2004; Ranganath et al., 2004a; Squire et al., 2007). In functional imaging studies, multiple factors, including the strength of the memory and the extent to which associated source details are recalled, influence hippocampal activity during episodic memory retrieval (Eldridge et al., 2000; Fortin et al., 2004; Ranganath et al., 2004b; Squire et al., 2007; Wais et al., 2010). Still, retrieval involves several subprocesses subserved by interacting cognitive modules (Moscovitch, 1992) and the specific contributions of these components to retrieval-related neural responses are not fully understood. For example, brain activity during retrieval may be modulated by the extent of task engagement, mental search, depth of processing, or post-retrieval monitoring. Regions activated during encoding can be engaged during retrieval of the original memory, suggesting that reactivation of the memory trace and associative re-encoding are additional retrieval sub-processes (Nyberg et al., 2000; Buckner et al., 2001; Woodruff et al., 2005). Distinct elements, such as memory strength and degree of search during memory retrieval attempts, may be highly correlated; studies intending to examine an isolated process may be confounded by these uncontrolled components. Additional investigation is necessary to better identify and understand the neural bases underlying subcomponents of memory retrieval.

Reports of relative signal differences between conditions, including increased activity with greater retrieval success, higher confidence, or more accurate source recollection (Yonelinas et al., 2005; Daselaar et al., 2006; Montaldi et al., 2006), have contributed significantly to our current understanding of hippocampal function during retrieval. While such comparisons are useful for distinguishing separable neural processes, they assume isolated insertion of independent components without accounting for interactions between sub-processes (Friston et al., 1996). For example, contrasting remembered with forgotten items during attempted recall 
yields functional responses often attributed to retrieval success. However, within this contrast are differences in attention or search, and associated task difficulty, that also vary with recall success or failure.

Examining the direction of blood oxygen level dependent (BOLD) signal deflection from baseline may help reveal functionally relevant deactivations otherwise masked by relative betweencondition differences. Interpreting a change from baseline carries certain challenges, such as determining which processes are active during the baseline state (Gusnard and Raichle, 2001) or whether deflection represents engagement of task-relevant or gating of task-irrelevant activity. However, information about the magnitude of signal deflection can provide additional insight into taskdependent responses. For example, the magnitude of deactivation from baseline may correlate with the degree of task engagement, task difficulty, or response time, relationships largely hidden when cognitive subtraction is used exclusively. Examining such characteristics of the task-evoked response, in conjunction with the signal difference from an optimally controlled condition, may therefore permit a more powerful interpretation of a BOLD effect.

Emerging evidence suggests a role for the hippocampus in multiple cortical networks that support encoding and retrieval. Several studies have found correlated activity between the hippocampus and regions involved in the default activation mode (Greicius et al., 2004; Buckner et al., 2008). This "default network" is commonly defined as a set of regions in medial prefrontal and posterior, lateral temporal, and inferior parietal cortex that are most active during passive resting states but can also be engaged by internally directed or personally relevant thought. Evidence that these regions are active during autobiographical memory (Andreasen et al., 1995; Maguire, 2001; Addis et al., 2004a,b; Spreng and Grady, 2010) suggests an overlap between neural systems underlying resting-state activity and memory consolidation or retrieval. This possibility is supported by studies showing that default network regions are modulated by the degree of encoding success or recall confidence (Daselaar et al., 2004, 2009; Kim, 2010), and that disrupted default network connectivity corresponds with agingrelated memory deficits (Andrews-Hanna et al., 2007). Although activation of these regions may support some aspects of memory consolidation or recall, successful execution of many demanding cognitive tasks correlates with default network deactivation (McKiernan et al., 2003; Fox et al., 2005; Buckner et al., 2008).

Functional magnetic resonance imaging (fMRI) measurements during successful retrieval likely reflect an integrated response to a series of interacting processes rather than activity during an isolated moment of memory access. Recall involves multiple stages including, for example, directed search for the memory from storage, successful retrieval, and working memory processes necessary to hold an item online while performing post-retrieval processing, such as making a judgment about the recalled item. Cognitive control and selective attention may contribute to top-down modulation of memory retrieval or to post-retrieval monitoring (Moscovitch, 1992; Buckner, 2003; Daselaar et al., 2008). Given the position of the hippocampus at the intersection of overlapping networks, hippocampal activity during memory retrieval may be concurrently regulated by any of these stages of memory processing. Task-related deactivation, such as that seen in default network regions, might also explain relative signal differences in the hippocampus. A prior study demonstrated robust hippocampal deactivation during cued recall and post-retrieval processing of visual paired associates (Israel et al., 2010), described as "elaborative associative recall." Externally directed thought and task difficulty are known to deactivate the default network (Greicius et al., 2003b; McKiernan et al., 2003; Vincent et al., 2008), and maintenance of an item in working memory can suppress hippocampal activity (Axmacher et al., 2007). It is therefore possible that either directed search effort prior to retrieval or post-retrieval working memory processing is a primary mediator of hippocampal activity during memory retrieval, rather than the retrieval event, itself.

The present study sought to isolate the mechanisms underlying hippocampal deactivation during elaborative associative recall. Event-related fMRI was used to investigate BOLD responses during cued recall and post-retrieval processing of previously studied word pairs relative to a non-memory classification task. Consistent with prior evidence of hippocampal deactivation during elaborative associative recall of paired visual objects (Israel et al., 2010), results confirmed that hippocampal deactivation occurs during elaborative associative recall of paired words, allowing further isolation and examination of the factors that modulate this suppression. To investigate the neural correlates underlying retrieval-related components of memory search, successful retrieval, and post-retrieval processing, we examined task conditions that had different levels of each. Contrasts between conditions were first performed to reveal relative signal differences afforded by traditional subtraction methods. Impulse response curves were then examined to evaluate the temporal dynamics of the BOLD response and signal deflection relative to baseline, and relationships with task performance and reaction times. By isolating activations associated with attempted retrieval, this study was able to dissociate a hippocampal response linked to memory search from those linked to retrieval success or post-retrieval processing.

\section{MATERIALS AND METHODS SUBJECTS}

Fifteen healthy, right-handed, English-speaking volunteers with normal or corrected vision from the University of California, San Diego (UCSD) community and surrounding areas participated in this study. All subjects gave informed written consent in accordance with criteria of the UCSD Institutional Review Board. Five subjects were excluded from further analysis due to an insufficient number of poorly remembered trials; data from the remaining 10 participants (seven male, mean age 27.2 \pm 3.0 years) are reported.

\section{STIMULI}

Stimuli were 128 English nouns ranging from one to four syllables. Half of the words represented living and half represented nonliving items. Words were combined into 64 pairs pseudo randomly to prevent unintentional semantic associations between words.

\section{EXPERIMENTAL DESIGN}

During a pre-scan learn-to-criterion study task, 64 word pairs were presented one at a time on a laptop. Subjects were instructed to 
remember each word pair. Each pair appeared for $3 \mathrm{~s}$, followed by a fixation cross for $1 \mathrm{~s}$ (Figure 1A). After study, subjects were given a self-paced cued recall test in which one word from each pair appeared and subjects verbally responded with the pair of the presented word. Forgotten pairs were repeated in subsequent study-test sequences until all pairs were correctly identified.

After a delay of approximately $20 \mathrm{~min}$, subjects completed a modified version of previously described recall and classify tasks (Israel et al., 2010; Seibert et al., 2011) during event-related fMRI data acquisition (Figure 1B). In each trial a black box and a colored box were presented for $1 \mathrm{~s}$. A previously studied word then appeared in one of the boxes for $1 \mathrm{~s}$. The green box surrounding the presented word served as a cue to classify the presented word as living or non-living (classify condition). A red box around the missing pair cued subjects to recall the pair of the presented word and classify the pair as living or non-living (recall condition). Subjects responded "living" or "non-living" in both tasks and were given a third response option of "unsure" in the recall task if they did not remember the pair of the presented word. Subjects were instructed to respond as quickly and accurately as possible using their right hand on a four-button response box. The cue boxes remained on the screen for $2 \mathrm{~s}$ following word presentation, and trials were jittered with $1.5-15 \mathrm{~s}$ of fixation baseline, calculated to optimize the study design for modeling the hemodynamic response to trials (Dale and Buckner, 1997; Dale, 1999). Each word appeared once and both words of a pair were assigned to the same condition (classify or recall). Equal numbers of classify and recall trials were pseudo randomly distributed across four runs each lasting $403 \mathrm{~s}$, and the two words comprising each pair were presented in different runs.

Following the scan, subjects completed a self-paced cued recall test (Figure 1C) to better evaluate retrieval success during the scanned recall test. One word from each pair was presented and subjects verbally reported the word's pair. Throughout the remainder of the manuscript, word pair associates correctly and incorrectly recalled during the post-scan recall test are respectively referred to as strongly remembered and poorly remembered.

\section{fMRI PARAMETERS}

Imaging was performed using a 3.0-T General Electric scanner at the UCSD Keck Center for Functional MRI. Functional data were acquired using a gradient-echo, echo-planar, T2*-weighted pulse sequence (time repetition, $\mathrm{TR}=2.5 \mathrm{~s}$; one shot per repetition; echo time $=30$; flip angle $=90^{\circ}$; bandwidth $=31.25 \mathrm{MHz}$ ). Each volume contained 40 slices oriented perpendicular to the long axis of the hippocampus with $3.4 \mathrm{~mm} \times 3.4 \mathrm{~mm} \times 4 \mathrm{~mm}$ voxels. Field maps were acquired to measure and correct for static field inhomogeneities (Smith et al., 2004). A T1-weighted structural scan was acquired in the same plane and of the same voxel size as the functional scans and a high resolution $(1 \mathrm{~mm} \times 1 \mathrm{~mm} \times 1 \mathrm{~mm}) \mathrm{T} 1-$ weighted anatomical scan was acquired using an inversion recovery prepared fast spoiled gradient recalled sequence providing high gray-white contrast for anatomical delineation.

\section{fMRI DATA ANALYSIS}

Functional data were corrected for spatial distortions using field maps (Smith et al., 2004). Using the AFNI suite of programs (Cox, 1996), data from each run were reconstructed and slices were temporally aligned and co-registered using a three-dimensional image alignment algorithm. A threshold mask of the functional data was applied to remove voxels outside the brain and separate functional runs were smoothed with a 4-mm FWHM Gaussian blur, corrected for motion, and concatenated. Anatomical scans and data output of the functional scans were normalized to Talairach

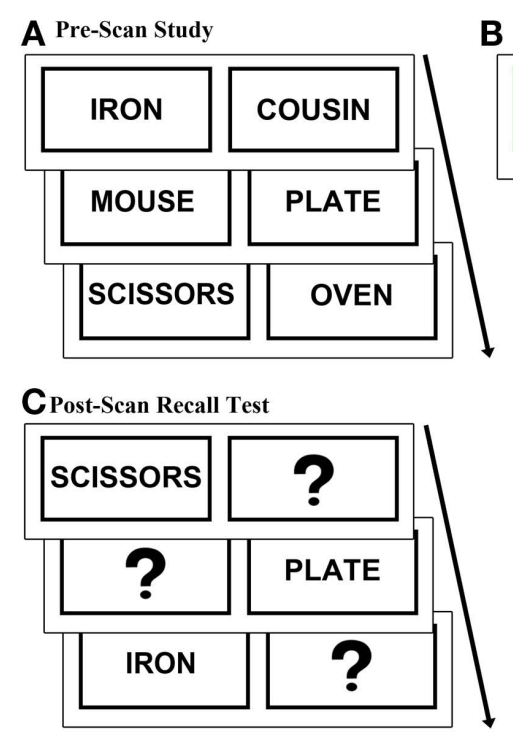

FIGURE 1 | Experimental design. (A) Prior to scanning, 64 word pairs were presented sequentially and subjects were instructed to memorize each pair. (B) During scanning, trials randomly alternated between classify and recall task conditions. In the classify task, a green box cued subjects to classify the

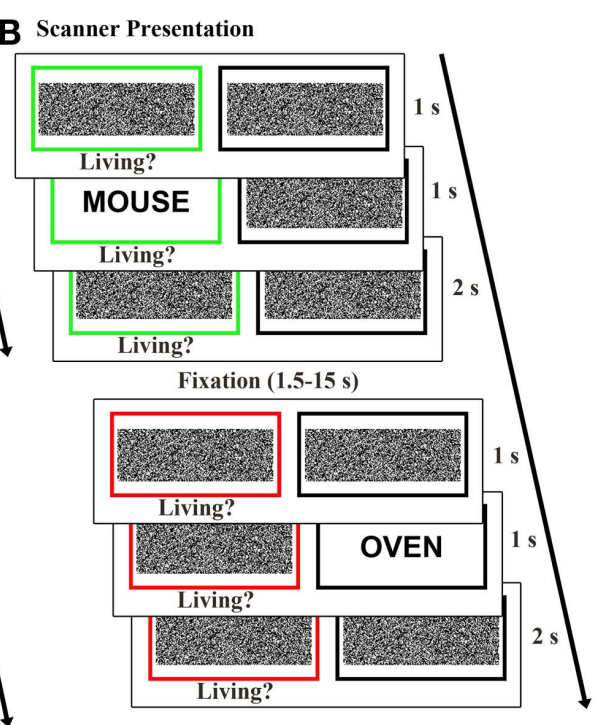

presented word as living or non-living. In the recall task a red box cued subjects to recall and classify the pair of the presented word. (C) In a post-scan recall test, subjects saw one word at a time from each pair and were asked to recall and vocally report the word's pair. 
space (Talairach and Tornoux, 1988) after standard landmarks were manually defined on the anatomical scans.

The region of interest large deformation diffeomorphic metric mapping (ROI-LDDMM) alignment technique was applied to improve alignment of the medial temporal lobe between subjects (Miller et al., 2005). Medial temporal lobe subregions, including bilateral hippocampus, perirhinal, entorhinal, and parahippocampal cortices, were defined for each subject on Talairach transformed images. Previously described landmarks were used to define perirhinal, entorhinal (Insausti et al., 1998), and parahippocampal (Stark and Okado, 2003) cortex. These anatomical regions of interest were aligned with a modified model of a previously created template segmentation (Kirwan et al., 2007) using ROI-LDDMM. Functional imaging data underwent the same ROILDDMM transformation to ensure alignment with each subject's anatomical data.

Multiple regression analysis was performed to generate general linear models for task conditions of interest. Each model included six motion regressors obtained from the registration process along with regressors for each condition. The hemodynamic response for the 17.5-s following the stimulus onset was estimated using signal deconvolution with TENT basis functions (Cox, 1996). Task regressors were included for three conditions: strongly remembered trials, defined as recall trials for which subjects correctly recalled the pair during the post-scan recall test; poorly remembered trials, defined as recall trials for which subjects incorrectly recalled or forgot the pair during the post-scan recall test; and correct classify trials. Three subtractions were computed to compare activity between (1) strongly remembered and classify, (2) poorly remembered and classify, and (3) poorly remembered and strongly remembered conditions. A conjunction of overlapping voxels from contrasts one and two was performed to identify voxels with greater activation or deactivation during strongly remembered and poorly remembered relative to classify.

To examine BOLD signal variation across subregions of the hippocampus, a structural mask that segmented the anatomically defined left and right hippocampus into eight $4 \mathrm{~mm}$ slices along the long axis was applied to each subject's data. Beta values for each $4 \mathrm{~mm}$ slice were extracted and a three-way ANOVA with factors of task (classify, strongly remembered, poorly remembered), hemisphere, and slice (eight slices, anterior to posterior) was performed.

The across-subject average impulse response was extracted for a hippocampal seed region of interest functionally defined from the conjunction analysis. The average impulse response curves for each of the three conditions (classify, strongly remembered, and poorly remembered) were used as model hemodynamic response functions. Multiple linear regression was used to estimate the fit of the hemodynamic response across the brain to these time-course models. Significant clusters in which the hemodynamic response fit the hippocampal model response across conditions were displayed on a statistical map overlaid onto an average structural image.

Amplitude-modulated regression was used to identify regions in which the hemodynamic response correlated with response time. Correlations were computed by examining the relationship between BOLD signal and response time on a trial by trial basis.
At each voxel, a general linear model was constructed with regressors for both the mean hemodynamic response and the correlation between BOLD signal and response time for the classify and recall conditions. Correlations from the classify and recall tasks were compared to identify regions in which the correlation strength depended on task condition.

Voxel-wise $t$-tests compared parameter estimates from the 7.5- to 12.5-s of each condition. The hemodynamic response was expected to have the greatest deflection from baseline during this time interval, based on impulse response curves from previous studies using a similar recall task (Israel et al., 2010; Seibert et al., 2011). For whole-brain analyses, significant clusters, including at least seven contiguous voxels $(p<0.01$, two-tailed and corrected for multiple comparisons), were displayed on a statistical map overlaid onto an average structural image. Applying a whole-brain correction for multiple comparisons can prevent detection of significant activations within a small region of interest selected a priori; therefore, significant hippocampal clusters were corrected for multiple comparisons within the hippocampus by including at least four contiguous voxels $(p<0.05$, two-tailed). Correction for multiple comparisons was performed using a Monte Carlo simulation in AFNI (afni.nimh.nih.gov/pub/dist/doc/program_help/3dClustSim.html), using a whole-brain functional volume (28,907 voxels) and a manually defined structural mask of the combined left and right hippocampus (139 voxels). The hemodynamic response function was then extracted for each condition within each cluster of interest and averaged across subjects.

\section{RESULTS}

\section{BEHAVIOR}

Subjects took an average ( \pm SE) of $3.5 \pm 0.4$ study runs to memorize all 64 word pairs. During scanning, classification accuracies were similar between classify trials and recall trials for which a classification was made [classify: $92 \pm 1 \%$, recall: $87 \pm 2 \%$; $t(9)=1.83$, $p>0.10]$. Subjects responded "unsure" to $15 \pm 2 \%$ of recall trials. Response times were faster in the classify than in the recall task [classify: $1271 \pm 60 \mathrm{~ms}$, recall: $2396 \pm 159 \mathrm{~ms} ; t(9)=8.40$, $p<0.001$ ]. Reaction times during the scanned test were analyzed based on accuracy during the post-scan recall test. There was no difference in reaction time between trials that were correctly versus incorrectly recalled during the post-scan test for the classify condition [strongly remembered classify: $1266 \pm 73 \mathrm{~ms}$, poorly remembered classify: $1422 \pm 151 \mathrm{~ms} ; t(9)=1.16, p=0.27$ ] and a trend toward a shorter reaction time for strongly versus poorly remembered pairs in the recall condition [strongly remembered: $2371 \pm 154 \mathrm{~ms}$, poorly remembered: $2504 \pm 207 \mathrm{~ms} ; t(9)=1.85$, $p<0.10]$. Post-scan recall was better for pairs that appeared in the recall than in the classify condition [recall: $83 \pm 4 \%$, classify: $73 \pm 7 \% ; t(9)=2.72, p<0.05]$.

\section{FUNCTIONAL MAGNETIC RESONANCE IMAGING Differential responses for strongly remembered, poorly remembered, and classify trials}

To evaluate consistency between these results and previous reports of BOLD signal changes during associative memory retrieval, a whole-brain analysis was performed using contrasts between 
the classify, strongly remembered (correct post-scan cued recall responses), and poorly remembered (incorrect post-scan cued recall responses) conditions. Results from this traditional subtraction analysis allowed further examination of the hemodynamic response and associated cognitive functions that form the bases for such prior findings. Activations were compared between the strongly remembered and classify conditions to identify regions more active during memory retrieval followed by post-retrieval processing compared to a control task (Figure 2A), and between poorly remembered and classify trials to investigate activity related to retrieval effort (Figure 2B). BOLD signals during poorly and strongly remembered trials were compared to examine unsuccessful memory retrieval efforts relative to successful memory retrieval and post-retrieval processing (Figure 2C). All contrasts were significant at the $p<0.01$ level and corrected for multiple comparisons as described in the section "fMRI Data Analysis". Regions with greater activity for poorly and strongly remembered than classify trials included left dorsolateral prefrontal cortex
(PFC), dorsal anterior cingulate cortex, anterior insula, and superior parietal cortex. Impulse response curves confirmed that BOLD signal was activated above fixation baseline to a greater extent for the poorly and strongly remembered than classify condition in these regions. The reverse relationship of reduced activity for poorly and strongly remembered relative to classify trials was observed in bilateral amygdala, medial PFC, precuneus, posterior cingulate cortex (PCC), and temporal cortex. Impulse response curves revealed that these differences were due to greater signal deflection below baseline for poorly and strongly remembered than classify trials. Activity was also reduced in left anterior hippocampus for strongly remembered compared to classify trials, consistent with prior findings (Israel et al., 2010). PCC and left inferior parietal cortex demonstrated less activation for the poorly remembered than strongly remembered condition. Impulse response curves illustrated greater negative signal deflection in these regions for poorly remembered compared to strongly remembered trials.

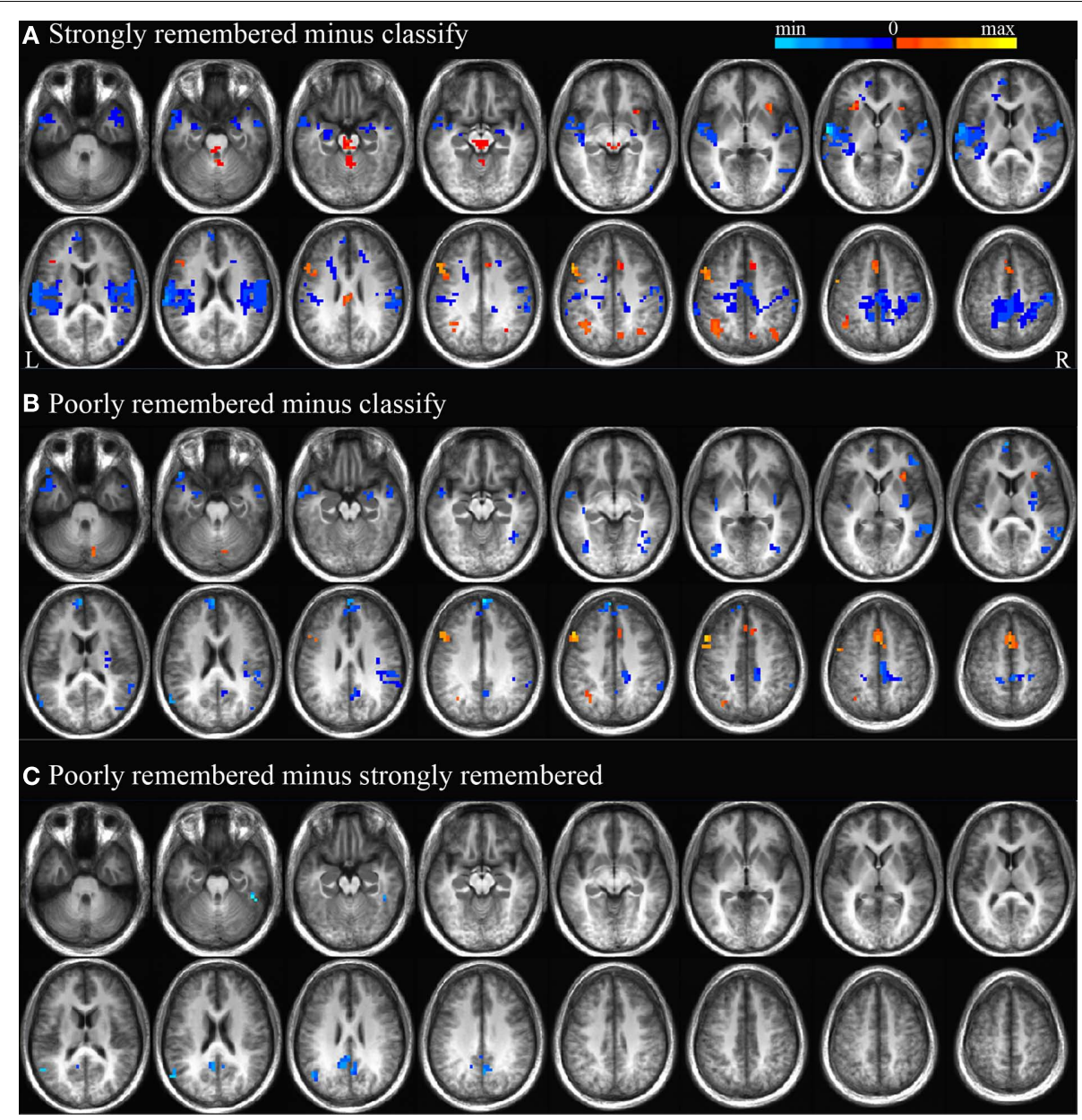

FIGURE 2 | Strongly remembered minus classify, poorly remembered minus classify, and poorly minus strongly remembered contrasts. Regions with significant BOLD signal differences $(p<0.01)$ between strongly remembered and classify trials (A), poorly remembered and classify trials (B), and poorly and strongly remembered trials $(\mathbf{C})$. Positive activity differences are displayed in warm colors and negative activity differences are displayed in cool colors. Subtraction maps are overlaid on every five axial slices of an average anatomical image of all subjects. As depicted in Figure 5A, these activation differences are expected to represent processing related to search, retrieval and post-retrieval processing $(\mathbf{A}, \mathbf{C})$, or search only $(\mathbf{B})$. 


\section{Anterior and posterior hippocampal activity}

Because of the small region of interest, a probability threshold of $p<0.05$ (corrected for multiple comparisons within the hippocampus as described in the section "fMRI Data Analysis") was used to examine BOLD signal changes in the hippocampus. Contrasts of strongly remembered versus classify, poorly remembered versus classify, and poorly versus strongly remembered trials were performed. These contrasts revealed less activation for strongly remembered than classify trials in bilateral anterior hippocampus, less activation for poorly remembered than classify trials in bilateral anterior and posterior hippocampus, and less activation for poorly than strongly remembered trials in left anterior and bilateral posterior hippocampus.

To identify regions in which activity was greater for both the poorly and strongly remembered than classify conditions, a conjunction analysis was used to identify the overlap of clusters more responsive during poorly remembered than classify and during strongly remembered than classify trials $(p<0.05)$. This conjunction yielded a cluster in left anterior hippocampus for which impulse response curves, relative to fixation baseline, demonstrated a stepwise decrease in activation from classify to strongly remembered to poorly remembered trials (Figure 3A). There was a main effect of task in this cluster $[F(2)=19.14$, $p<0.001]$ and pair-wise comparisons confirmed greater deactivation for poorly remembered than classify $[t(9)=4.63, p<0.005]$, for strongly remembered than classify $[t(9)=4.42, p<0.005]$ and for poorly remembered than strongly remembered trials $[t(9)=3.67, p<0.01]$. A cluster was also identified that survived whole-brain correction and extended into right anterior hippocampus, but the hippocampal portion of this cluster did
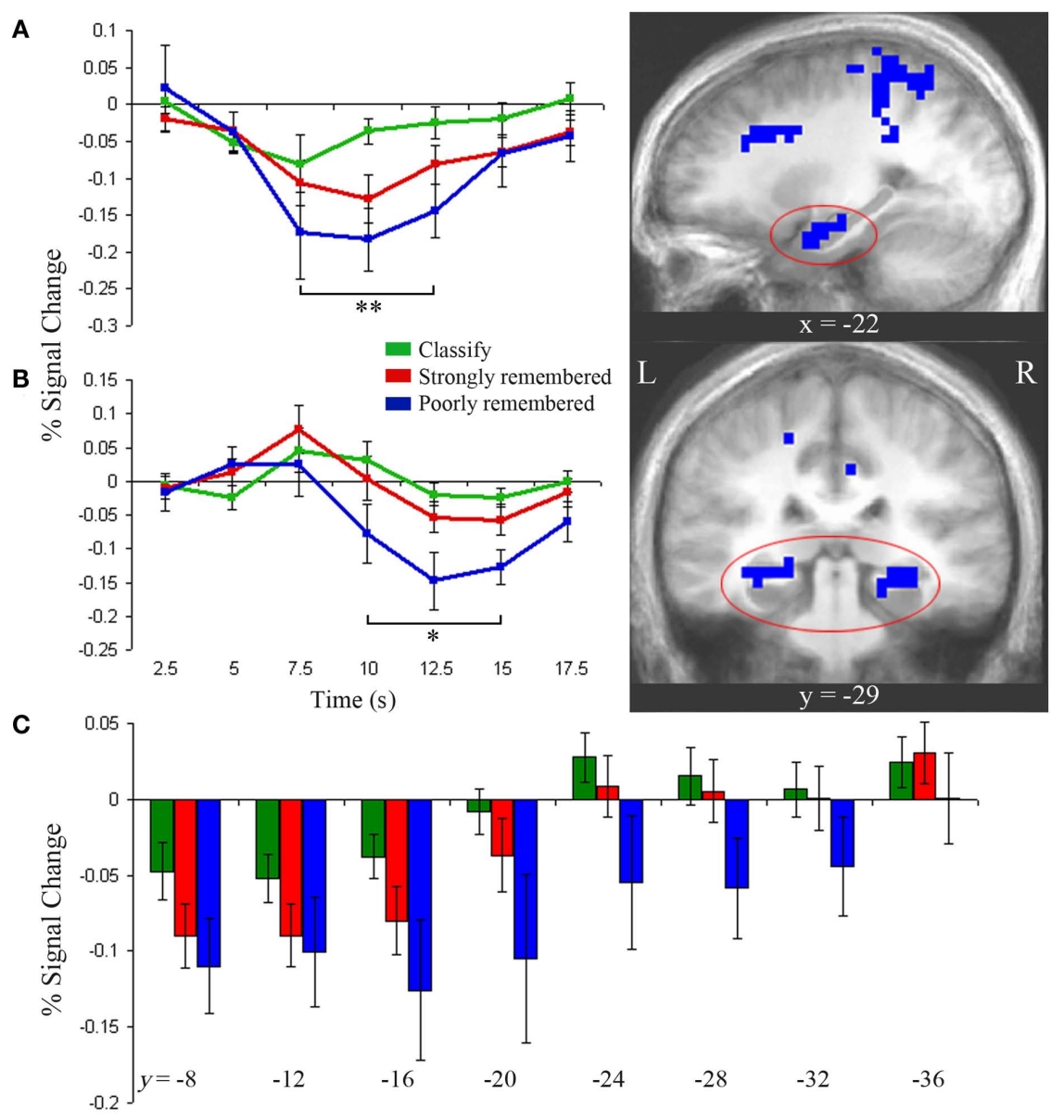

FIGURE 3 | Anterior and posterior hippocampal activity. (A) Left anterior hippocampus showed greater deactivation during strongly and poorly remembered relative to classify trials (right, $p<0.05$ ). Impulse response curves (left) illustrate a graded pattern of deactivation from fixation baseline greatest for poorly remembered (blue), intermediate for strongly remembered (red), and minimal for classify (green) trials. **Indicates poorly remembered < classify, strongly remembered < classify, and poorly remembered $<$ strongly remembered (paired $t$-tests, $p<0.01$ ). (B) Bilateral posterior hippocampus was more active for strongly versus poorly remembered trials $(p<0.05)$. Impulse response curves reveal late deactivation for poorly remembered trials only. ${ }^{*}$ Indicates poorly remembered $<$ classify and poorly remembered $<$ strongly remembered (paired $t$-tests, $p<0.01$ ). (C) Bilateral hippocampus showed a gradient of decreasing deactivation, from 7.5 to $12.5 \mathrm{~s}$, from anterior to posterior regions. Each hippocampal subregion represents a 4-mm thick slice along the long axis of the hippocampus. Error bars represent SE of the mean. Subtraction maps are overlaid on sagittal (A) and coronal (B) slices of the average anatomical image of all subjects. 
not survive correction for multiple comparisons within the hippocampus.

Impulse response curves from the posterior hippocampal clusters identified in the poorly remembered versus strongly remembered contrast were examined and revealed a delayed posterior response compared to the anterior response. Posterior hippocampus showed a late-onset response with negative deflection from baseline for the poorly remembered condition (Figure 3B). Although these clusters were identified using the 7.5- to 12.5-s response interval selected a priori, the time-course of the response prompted additional post hoc analysis of early and late time-points. A main effect of task was observed between 10 and $15 \mathrm{~s}[F(2)=12.17, p<0.001]$, driven by deactivation for poorly remembered trials relative to strongly remembered trials $[t(9)=4.14, p<0.005]$ and classify trials $[t(9)=3.46, p<0.01]$. At $7.5 \mathrm{~s}$, a trend for early positive activation above baseline was observed during the strongly remembered condition $[t(9)=2.16$, $p=0.06]$, but responses did not differ between task conditions $(p=0.31)$.

\section{Hippocampal activation gradient}

Figure 3 illustrates the distinct contributions of anterior and posterior hippocampal regions to poorly and strongly remembered trial responses. However, to directly examine how hippocampal activity varies along the anterior-posterior axis, left, and right hippocampus were each segmented into eight slices perpendicular to the long axis and beta values for each condition were extracted in each slice. No hemispheric differences were found $(p=0.78)$, but main effects of task $[F(2,18)=3.64, p<0.05]$ and slice $[F(7,63)=13.10, p<0.001]$ and a task by slice interaction $[F(14,126)=1.99, p<0.05]$ were observed. Deactivation decreased along an anterior to posterior gradient across tasks (Figure 3C). Anterior regions showed deactivation below baseline during all conditions $[y=-7$ to -18 , one-sampled $t$-tests: classify, $t(9)=3.03, p<0.05$; strongly remembered, $t(9)=4.27$, $p<0.005$; poorly remembered, $t(9)=3.00, p<0.05$ ] and a main effect of task [ $y=-15$ to $-22: F(2)=3.85, p<0.05]$, reflecting increasing deactivation from classify to strongly remembered to poorly remembered conditions. In contrast, posterior regions $(y=-27$ to -34$)$ only showed deactivation in the poorly remembered condition, with a main effect of task $[F(2)=5.11, p<0.05]$ driven by greater deactivation for poorly remembered relative to classify $[t(9)=2.29, p<0.05]$ and to strongly remembered trials $[t(9)=2.74, p<0.05]$.

\section{Similar activation patterns in anterior hippocampus and default network}

Results suggest that responses in the anterior hippocampus are modulated by both task and memory strength. To examine the effects of task and memory strength in the whole-brain we identified regions in which the time-course of the hemodynamic response matched that in anterior hippocampus. For each voxel and for each condition, the hemodynamic response was fit to the impulse response curves previously identified in left anterior hippocampus (Figure 3A). The activity time-courses in medial PFC, PCC, left inferior parietal cortex, and temporal pole were modeled by the seed hippocampal response across all conditions $(p<0.001$; Figure 4). Impulse response curves illustrated stepwise deactivation from classify to strongly remembered to poorly remembered conditions. The average response across these regions differed across task conditions $[F(2)=11.90, p<0.001]$, confirming greater deactivation for poorly remembered than classify $[t(9)=3.86, p<0.005]$, for poorly remembered than strongly remembered $[t(9)=4.09, p<0.005]$, and a trend for greater deactivation for strongly remembered than classify trials $[t(9)=2.17$, $p=0.06]$. In addition, dorsal anterior cingulate cortex and anterior insula fit the inverse of the response model. Impulse response curves for these clusters demonstrated task-positive activation for all conditions, with greater positive deflection above baseline for poorly remembered and strongly remembered trials than classify trials.

\section{Activity correlations with response time}

To examine a possible relationship between response fluency and hippocampal and default network suppression, correlations between BOLD signal and response time were computed. Activity was negatively correlated with response time during both the classify and recall tasks in bilateral anterior hippocampus, precuneus, PCC $(p<0.01)$, medial PFC, and superior temporal cortex $(p<0.001)$. Thus, greater activity in these regions was correlated with a faster response time. Activity in these regions was more strongly correlated with response time for the recall task than for the classify task $(p<0.01)$.

\section{DISCUSSION}

In the present study, negative BOLD signal deflection was observed in anterior hippocampus during the attempted recall of both strongly and poorly remembered word pair associates, and this deactivation was greatest for poorly remembered associations. The response in anterior hippocampal regions was distinct from that in posterior regions, which showed a late-onset deactivation only during the poorly remembered condition. A model of the hemodynamic response in anterior hippocampus was fit to the whole-brain, and a similar pattern of graded deactivation across task conditions was identified in regions associated with the default network. Finally, response times were inversely correlated with BOLD signal in anterior hippocampus and default network regions, and this correlation was stronger in the recall than classify task.

\section{ANTERIOR HIPPOCAMPUS DEACTIVATES DURING ATTEMPTED MEMORY RETRIEVAL}

Deactivation of hippocampal subregions may appear paradoxical in light of numerous studies reporting greater activity in anterior (Gabrieli et al., 1997; Cansino et al., 2002; Dobbins et al., 2003), posterior (Daselaar et al., 2006; Montaldi et al., 2006), or global (Nyberg et al., 1996; Eldridge et al., 2000; Henson, 2005; Eichenbaum et al., 2007) hippocampus for recollection versus familiarity, retrieval with increased confidence, or old versus new judgments. Relative signal differences may be particularly susceptible to ambiguous interpretations, since they may be generated by either the more positively activating or negatively deactivating condition. Although relative signal increases during successful 


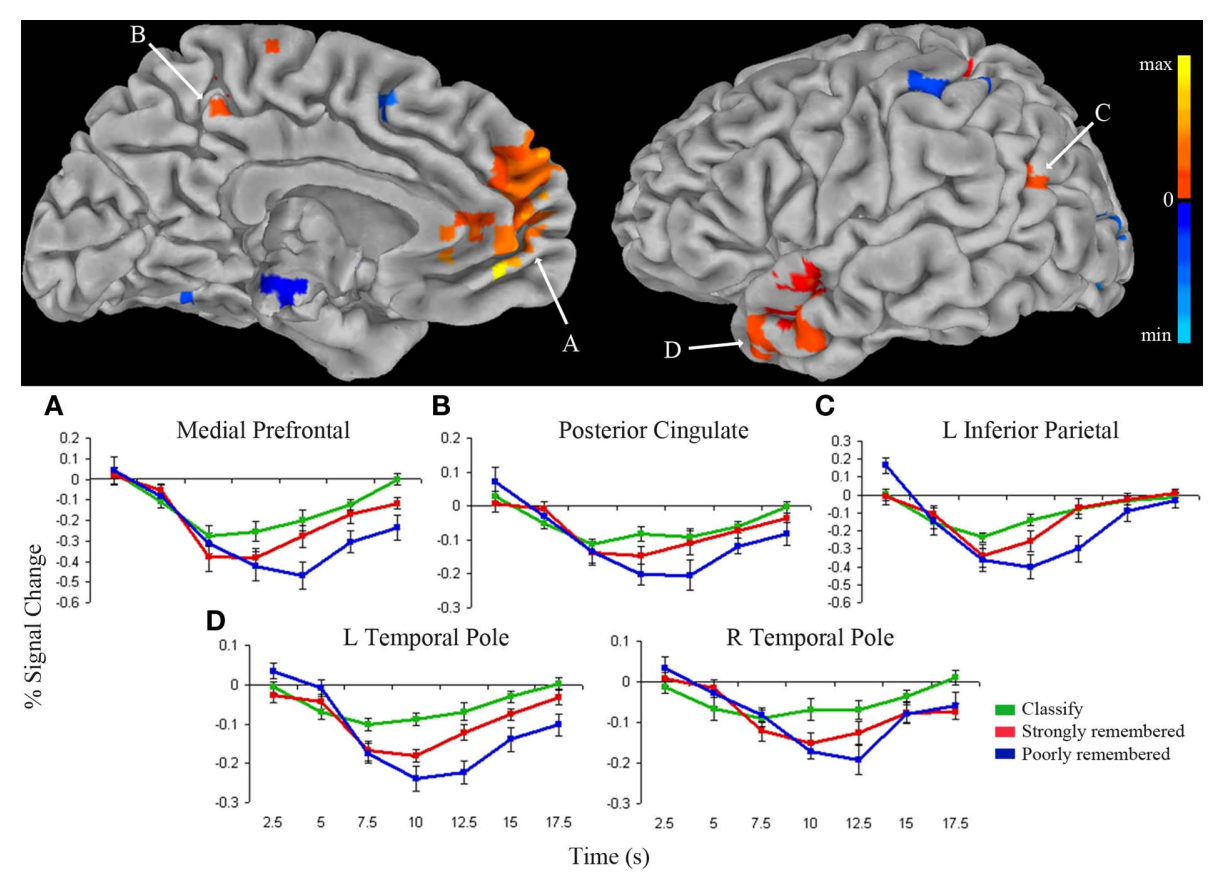

FIGURE 4 | Regions fitting the anterior hippocampus response model. Clusters that directly fit the response model (warm colors) or fit the inverse of the response model (cool colors) for the classify, strongly remembered, and poorly remembered conditions are overlaid on the left medial (top left) and lateral (top right) pial surface of the Talairach and Tournoux N27 average brain $(p<0.001)$. Color intensity represents strength of fit with the anterior hippocampal impulse response model presented in Figure 3A. The average impulse response curves (bottom) from the medial prefrontal cortex (A), posterior cingulate (B), left inferior parietal cortex (C), and left and right temporal pole (D) show decreased activity below fixation baseline for classify (green), followed by greater deactivation for strongly remembered (red), and greatest deactivation for poorly remembered (blue). versus unsuccessful memory recall may reflect retrieval-driven activations, an alternative explanation might be that task-relevant deactivations contribute to such signal changes. The present results support a growing body of evidence that anterior or posterior hippocampus can deactivate during tasks that would otherwise be expected to engage the hippocampus, including retrieval of spatial memories (Rekkas et al., 2005) or visual paired associates (Israel et al., 2010), or during configural associative learning (Meltzer et al., 2008). Together, these findings highlight the possibility that both positively and negatively activating processes during retrieval provide task-relevant contributions to BOLD signal differences.

The current findings help to disentangle the contributions of memory search, retrieval success, and post-retrieval processing to hippocampal responses during retrieval. In the recall task, memory search was encouraged by the instruction to recall a paired associate, retrieval success was assessed by post-scan recall accuracy, and post-retrieval processing included a classification judgment about the recalled word. Figure 5A presents a model of the hypothetical cognitive processes involved during successful and unsuccessful performance of this task. In contrast with poorly remembered trials, during which retrieval and post-retrieval processes are markedly reduced or absent, strongly remembered trials consistently involve these operations. Both strongly and poorly remembered trials engage search, as directed by the task, although poorly remembered trials are primarily comprised of search. All three of these retrieval-specific processes are minimal during the classify task which does not require recall. The anterior hippocampal deactivation observed during both strongly and poorly remembered trials therefore appears to be associated with processes involved in memory search.

Studies that examined impulse responses have shown evidence for task-positive global (Eldridge et al., 2000; Wais et al., 2010) or regional (Gimbel and Brewer, 2011) hippocampal activation during retrieval, suggesting that either recollection or its associated processes increase hippocampal activation above baseline or pre-task levels. However, given the known function of the hippocampus in encoding the ongoing stream of experience, another consideration is how retrieval efforts might influence tonic encoding-related activity in the hippocampus. Encoding processes are known to occur during retrieval (Nyberg et al., 2000; Buckner et al., 2001) and many reports suggest that successful encoding (Lepage et al., 1998; Sperling et al., 2003) or novelty (Strange et al., 1999; Daselaar et al., 2006) engages anterior hippocampus. Findings from a recent word recognition study (Huijbers et al., 2009) indicate that incidental encoding can occur concurrently with intentional retrieval and that these operations may compete for shared neural resources in the medial temporal lobe. Figure 5B illustrates how task conditions might regulate hippocampal activity under the alternate assumptions that the subregion is engaged by either encoding or recall. Activity modulated by recall should increase during strongly remembered associations only. However, tonic encoding-related activity in the hippocampus may also be reduced by retrieval efforts regardless of retrieval success, as attention and neural resources are directed 
A Component retrieval processes

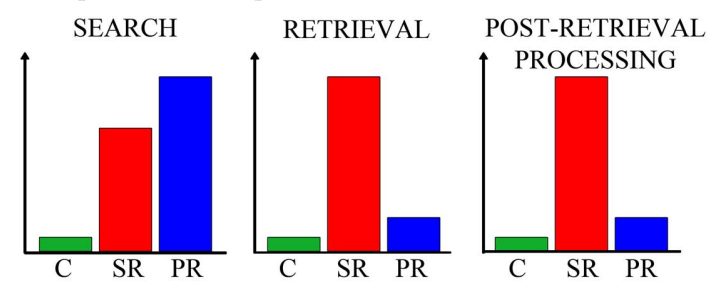

B Hippocampal encoding and recall activity

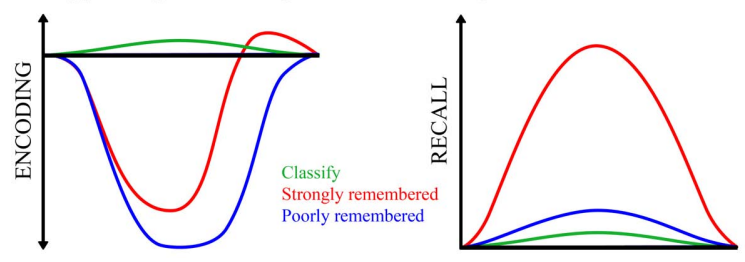

FIGURE 5 | Cognitive and neural processing models. (A) The hypothetical cognitive processes involved in an elaborative associative retrieval task, and the magnitude to which each task condition engages these processes, are depicted. The $y$-axis represents arbitrary units measuring degree of engagement. From this model, the following cognitive processes are expected to result from task condition contrasts: If engaged more for strongly remembered than classify, the process(es) might be search, retrieval, or post-retrieval processing; if engaged more for poorly remembered than classify and for poorly than strongly remembered, the process might be search. C, classify; SR, strongly remembered; PR, poorly remembered. (B) Hippocampal activity for each task condition is illustrated, under alternative models for encoding- and recall-mediated responses. Both strongly and poorly remembered trials are hypothesized to reduce encoding-related activity (left). Strongly remembered trials are expected to increase recall-related activity (right). The $x$-axes and $y$-axes are in arbitrary units of time and neural activity, respectively.

away from encoding. The magnitude or duration of this searchdriven, hippocampal disengagement may be greatest for unsuccessful retrieval attempts, where search comprises the entirety of the trial. Hippocampal disengagement would be intermediate for successful recall trials, which on average are comprised of brief search followed by retrieval success and post-retrieval processing, the latter of which evoke re-engagement of encoding processes as the retrieved and processed material is re-encoded. Thus, a task-positive hippocampal response for successful retrieval would not be inconsistent with the proposed model that hippocampal activity is primarily driven by encoding and not retrieval, especially if the task-positive responses occur under conditions where search processes are minimal. In most fMRI studies of episodic retrieval, recognition tasks are used, which would not typically engage guided search to the extent of cued recall.

\section{RETRIEVAL-RELATED ANTERIOR AND POSTERIOR HIPPOCAMPAL RESPONSES ARE DISTINCT}

Hippocampal response patterns across task conditions were both spatially and temporally distinct. This dissociation is consistent with evidence from rodent and human studies of subregion specialization within the hippocampus which suggests that the structure does not function as a single unit. Prior human neuroimaging studies provide additional support for the anterior to posterior functional gradient observed in the current study, reporting that anterior and posterior regions respectively subserve encoding (Strange et al., 1999; Sperling et al., 2003) and retrieval (Lepage et al., 1998; Daselaar et al., 2006) or that both encoding and retrieval functions may be posteriorly localized (Greicius et al., 2003a). Higher resolution functional imaging within the medial temporal lobe has allowed delineation of anatomical hippocampal subregions that perform distinct functions, including dentate gyrus/CA3 specialization for encoding or pattern separation, CA1/subiculum specialization for retrieval or pattern completion (Eldridge et al., 2005; Bakker et al., 2008; Lacy et al., 2011; Suthana et al., 2011), and distinct roles of anterior and posterior CA1 in match/mismatch detection (Duncan et al., 2011). Such findings complement an expanding body of animal lesion and electrophysiology literature reporting unique contributions of hippocampal subfields to encoding, consolidation and retrieval, pattern completion and pattern separation, or different forms of temporal, spatial, episodic, or working memory (Kesner et al., 2004; Lee and Kesner, 2004; Daumas et al., 2005; Hoge and Kesner, 2007; Leutgeb et al., 2007; Gilbert and Brushfield, 2009).

In the current study, deactivation during poorly remembered trials extended throughout the hippocampus, suggesting that similar neural mechanisms are at play in anterior and posterior subregions during failed retrieval efforts. However, the temporal lag of the posterior hippocampal deactivation may indicate regional delay of neural processing, an effect of the vascular anatomy and hemodynamic response, or a mixing of a modest positive response component with a more general and robust negative response component. The trend for an early posterior hippocampal task-positive activation could possibly be related to retrieval itself, to encoding of the stimulus cue, or to episodic re-encoding that accompanies retrieval events (see Figure 5B; Buckner et al., 2001; Stark and Okado, 2003; Kirwan and Stark, 2004; Gimbel and Brewer, 2011). The presence of this peak early in the signal timecourse and a lack of difference across task conditions suggest that it may reflect regional involvement of the posterior hippocampus in early task processing stages, such as encoding the word cue. Nevertheless, examination of the impulse response curves demonstrates that the robust difference between activations related to strongly and poorly remembered trials is primarily driven by suppression during poorly remembered trials rather than deviation from baseline for strongly remembered trials, at least in this task involving cued recall.

\section{SIMILAR ACTIVITY PATTERNS IN THE DEFAULT NETWORK AND ANTERIOR HIPPOCAMPUS}

Similar hemodynamic responses were elicited in anterior hippocampus and classic default network regions (medial PFC, precuneus, inferior parietal cortex, and temporal pole), consistent with documented functional correlations between the hippocampus and default network (Greicius et al., 2004; Buckner et al., 2008). Default network function is a topic of active exploration, as it remains unclear to what degree these regions are regulated by specific thoughts or external stimulation. Default network activity is believed to reflect passive, task-irrelevant processing; this network inversely correlates with "task-positive" regions and attenuates in response to goal-directed behavior or externally focused 
thoughts (Raichle et al., 2001; McKiernan et al., 2003; Fox et al., 2005). The default network may be sensitive to task performance in general, which should be considered as a potential factor underlying the hippocampal suppression observed in this study. However, areas deactivated during non-memory task performance only partially overlap with regions deactivated during elaborative memory retrieval, which have been found to include selective medial temporal regions (Israel et al., 2010). Components of overlapping default and memory networks may therefore be differentially regulated by general mental engagement and domain-specific task parameters, and broadly defined task-induced deactivations may not fully account for suppressed activity in the hippocampus.

While the functional significance of neural deactivation is a subject of ongoing investigation, it has been proposed that some deactivations are task-independent and arise when a goal-directed behavior attenuates a sustained level of resting-state activity (Shulman et al., 1997; Mazoyer et al., 2001; Raichle et al., 2001). This interpretation for task-induced default network deactivations may explain the decreased activity in regions of the default network observed in the present study. Alternatively, deactivations may be task-specific and represent reallocation of resources from taskirrelevant to task-critical regions (Drevets et al., 1995; Kawashima et al., 1995; McKiernan et al., 2003). This hypothesis provides a rationale for task-induced hippocampal suppression which may prevent interference with processes critical to the early stages of memory retrieval. While the function of the default network is less understood, the hippocampus is known to be highly specialized for associative encoding, which may compete with other functions important for efficient memory recall.

A recent associative memory fMRI study (Huijbers et al., 2011) demonstrated that the hippocampus and default network are coupled during retrieval but become uncoupled during encoding, independent of whether a memory task demands internally or externally oriented attention. Although the present results expand upon these findings to reveal correlated activity between the anterior hippocampus and default network during attempted retrieval, it remains unclear whether search or concurrent correlated operations directly underlie default-hippocampal coupling. Whereas default activity may be suppressed by a variety of task demands, the hippocampus may be less sensitive to non-memory task engagement or general task difficulty (Israel et al., 2010; Gimbel and Brewer, 2011). During a retrieval attempt, the hippocampus may transition accordingly to a state in which hippocampal encoding functions are minimized in favor of retrieval efficacy, while the default network is simultaneously deactivated by correlated task effort. Since episodic search should also be required in the cued source retrieval paradigm employed by Huijbers et al. (2011), further exploration is required to determine if hippocampal-default correlations are maintained during spontaneous retrieval with minimal search demands.

Neural deactivations have been associated with high working memory or attentional demands (Greicius et al., 2003b). While such processes are needed to perform the directed search and post-retrieval classification required in the current experiment, evidence is inconclusive as to whether these factors may contribute to hippocampal suppression. Although a study of autobiographical memory recall did not report a hippocampal response to elaboration following recall (Daselaar et al., 2008), the present authors have observed anterior hippocampal deactivation during elaborative retrieval of visual paired associates (Israel et al., 2010) and greater anterior hippocampal deactivation during recollection with post-retrieval classification compared to non-elaborative recollection (Gimbel and Brewer, unpublished observations). In the current experiment post-retrieval processing would not likely be initiated following unsuccessful recall. That deactivation occurred during poorly remembered trials suggests that this suppression may be specific to the search stages of memory retrieval. Regardless of retrieval success, search processes are presumably engaged during recall of both strong and poor memories, and retrieval efforts for weaker memories may require increased search. The stepwise modulation of deactivation in anterior hippocampus from classify to strongly remembered to poorly remembered trials supports the interpretation that this response may be associated with search. While post-retrieval judgments may further impact the hippocampus or default network, it appears likely that pre-retrieval search processes primarily drive the observed deactivation.

\section{BOLD SIGNAL IN THE HIPPOCAMPUS AND DEFAULT NETWORK INVERSELY CORRELATES WITH RESPONSE TIME}

Neural responses to subcomponents of retrieval, such as searching for episodic information, may be influenced by response fluency (Herron, 2007), which can roughly be gaged by response time. The present study found a negative relationship between response fluency and deactivation in the hippocampus and default network; in other words, longer response times or less fluent responses were associated with greater deactivation. Furthermore, this correlation was strengthened when the task additionally required memory recall. Assuming an absence of retrieval attempts during the classify task, the dependence of deactivation on response fluency in this control task suggests that general task difficulty or effort may regulate default network suppression. Nevertheless the correlation was much weaker in the control task and, indeed, despite instructions not to recall during the classify task, inadvertent memory search may have contributed to some degree of correlation in the classify condition. Such task-related differences in correlation strength support an interpretation that this deactivation could be modulated by task-specific retrieval fluency. Prior evidence indicates that memory strength may influence default network independent of task difficulty (Gimbel and Brewer, 2011). The present study extends this finding to suggest that effortful search, which may inversely correlate with memory strength, involves the concomitant suppression of the anterior hippocampus and default network.

\section{LIMITATIONS}

In the current study, hippocampal activation was reduced during task conditions involving guided memory retrieval effort. Although this response appears attributable to search processes and may reflect interactions between hippocampal encoding and retrieval functions, this interpretation relies on several assumptions that deserve consideration. First, the classify, strongly remembered, and poorly remembered trials are assumed to respectively involve minimal, moderate, and high levels of memory 
search. Although participants were explicitly instructed not to recall during classify trials, it is possible that incidental recall occurred during some classify trials. However, the significantly faster response times and lower post-scan recall accuracy for classify than recall trials suggest that subjects indeed performed the tasks as instructed.

Second, only strongly remembered trials are assumed to involve recollection and post-retrieval processing. Due to practical limitations, cued recall was not tested during scanning and post-scan recall was instead used to approximate recall success. It is possible that post-scan recall did not correspond exactly with retrieval performance during the scanned recall test; however, pairs remembered during the post-scan test were classified with high accuracy $(82 \pm 3 \%)$ during scanning, and there was a trend $(p=0.10)$ for slower reaction times during scanning for pairs that were forgotten than remembered during the post-scan test. These behavioral findings support the assumption that pairs recalled and forgotten after scanning were strongly and poorly remembered during the scanned recall test.

Finally, since it was not feasible to directly evaluate encoding during the current recall task, it remains unclear whether encoding-related activations in fact depended on search demands. Further investigation is warranted to determine how processes such as simultaneous encoding of the external sensory environment, of the stimulus cue, or re-encoding of recalled associations,

\section{REFERENCES}

Addis, D. R., Mcintosh, A. R., Moscovitch, M., Crawley, A. P., and Mcandrews, M. P. (2004a). Characterizing spatial and temporal features of autobiographical memory retrieval networks: a partial least squares approach. Neuroimage 23, 1460-1471.

Addis, D. R., Moscovitch, M., Crawley, A. P., and Mcandrews, M. P. (2004b). Recollective qualities modulate hippocampal activation during autobiographical memory retrieval. Hippocampus 14, 752-762.

Andreasen, N. C., O’leary, D. S., Cizadlo, T., Arndt, S., Rezai, K., Watkins, G. L., Ponto, L. L., and Hichwa, R. D. (1995). Remembering the past: two facets of episodic memory explored with positron emission tomography. Am. J. Psychiatry 152, 1576-1585.

Andrews-Hanna, J. R., Snyder, A. Z., Vincent, J. L., Lustig, C., Head, D., Raichle, M. E., and Buckner, R. L. (2007). Disruption of large-scale brain systems in advanced aging. Neuron 56, 924-935.

Axmacher, N., Mormann, F., Fernandez, G., Cohen, M. X., Elger, C. E., and Fell, J. (2007). Sustained neural activity patterns during working memory in the human medial temporal lobe. J. Neurosci.27, 7807-7816.

Bakker, A., Kirwan, C. B., Miller, M., and Stark, C. E. (2008). Pattern

are modulated by search or other aspects of episodic memory retrieval.

\section{CONCLUSION}

The present study reports deactivation of anterior hippocampus and default network regions during elaborative verbal episodic memory retrieval, and suggests that effortful search may underlie this deactivation. Anterior and posterior hippocampus functionally dissociated during this task, suggesting that separate hippocampal subregions may coordinate with distinct networks subserving different neural processes. Further investigation will help clarify whether search- and task-difficulty-related deactivations are dissociable phenomena and whether they are differentially linked to tonic and concurrent episodic encoding. These results shed light on both the complex factors regulating hippocampal engagement or disengagement and the functional significance of hippocampus-default network interactions during episodic memory retrieval.

\section{ACKNOWLEDGMENTS}

This work was supported by NINDS K02 NS067427, the General Electric Medical Foundation, and the University of California, San Diego Department of Neurosciences. Jena B. Hales is supported by the National Science Foundation through the Graduate Research Fellowship Program.

familiarity, and novelty. J. Neurophysiol. 96, 1902-1911.

Daselaar, S. M., Prince, S. E., and Cabeza, R. (2004). When less means more: deactivations during encoding that predict subsequent memory. Neuroimage 23, 921-927.

Daselaar, S. M., Prince, S. E., Dennis, N. A., Hayes, S. M., Kim, H., and Cabeza, R. (2009). Posterior midline and ventral parietal activity is associated with retrieval success and encoding failure. Front. Hum. Neurosci. 3:13. doi:10.3389/neuro.09.013.2009

Daselaar, S. M., Rice, H. J., Greenberg, D. L., Cabeza, R., Labar, K. S., and Rubin, D. C. (2008). The spatiotemporal dynamics of autobiographical memory: neural correlates of recall, emotional intensity, and reliving. Cereb. Cortex 18, 217-229.

Daumas, S., Halley, H., Frances, B., and Lassalle, J. M. (2005). Encoding, consolidation, and retrieval of contextual memory: differential involvement of dorsal CA3 and CA1 hippocampal subregions. Learn. Mem. 12, 375-382.

Dobbins, I. G., Rice, H. J., Wagner, A. D. and Schacter, D. L. (2003). Memory orientation and success: separable neurocognitive components underlying episodic recognition. $\mathrm{Neu}$ ropsychologia 41, 318-333.

Drevets, W. C., Burton, H., Videen, T. O., Snyder, A. Z., Simpson, J. R. Jr., and Raichle, M. E. (1995). Blood flow changes in human somatosensory cortex during anticipated stimulation. Nature 373, 249-252.

Duncan, K., Ketz, N., Inati, S. J., and Davachi, L. (2011). Evidence for area CA1 as a match/mismatch detector: a high-resolution fMRI study of the human hippocampus. Hippocampus. doi: 10.1002/hipo.20933. [Epub ahead of print].

Eichenbaum, H. (2004). Hippocampus: cognitive processes and neural representations that underlie declarative memory. Neuron 44, 109-120.

Eichenbaum, H., Yonelinas, A. P., and Ranganath, C. (2007). The medial temporal lobe and recognition memory. Annu. Rev. Neurosci. 30, 123-152.

Eldridge, L. L., Engel, S. A., Zeineh, M. M., Bookheimer, S. Y., and Knowlton, B. J. (2005). A dissociation of encoding and retrieval processes in the human hippocampus. J. Neurosci. 25, 3280-3286.

Eldridge, L. L., Knowlton, B. J., Furmanski, C. S., Bookheimer, S. Y. and Engel, S. A. (2000). Remembering episodes: a selective role for the hippocampus during retrieval. Nat. Neurosci. 3, 1149-1152.

Fortin, N. J., Wright, S. P., and Eichenbaum, H. (2004). Recollection-like memory retrieval in rats is dependent on the hippocampus. Nature 431, 188-191. 
Fox, M. D., Snyder, A. Z., Vincent, J. L., Corbetta, M., Van Essen, D. C., and Raichle, M. E. (2005). The human brain is intrinsically organized into dynamic, anticorrelated functional networks. Proc. Natl. Acad. Sci. U.S.A. 102, 9673-9678.

Friston, K. J., Price, C. J., Fletcher, P., Moore, C., Frackowiak, R. S., and Dolan, R. J. (1996). The trouble with cognitive subtraction. Neuroimage 4 , 97-104.

Gabrieli, J. D., Brewer, J. B., Desmond, J. E., and Glover, G. H. (1997). Separate neural bases of two fundamental memory processes in the human medial temporal lobe. Science 276, 264-266.

Gilbert, P. E., and Brushfield, A. M. (2009). The role of the CA3 hippocampal subregion in spatial memory: a process oriented behavioral assessment. Prog. Neuropsychopharmacol. Biol. Psychiatry 33, 774-781.

Gimbel, S. I., and Brewer, J. B. (2011). Reaction time, memory strength, and fMRI activity during memory retrieval: Hippocampus and default network are differentially responsive during recollection and familiarity judgments. Cogn. Neurosci. 2, 19-23.

Greicius, M. D., Krasnow, B., BoyettAnderson, J. M., Eliez, S., Schatzberg, A. F., Reiss, A. L., and Menon, V. (2003a). Regional analysis of hippocampal activation during memory encoding and retrieval: fMRI study. Hippocampus 13, 164-174.

Greicius, M. D., Krasnow, B., Reiss, A. L., and Menon, V. (2003b). Functional connectivity in the resting brain: a network analysis of the default mode hypothesis. Proc. Natl. Acad. Sci. U.S.A. 100, 253-258.

Greicius, M. D., Srivastava, G., Reiss, A. L., and Menon, V. (2004). Defaultmode network activity distinguishes Alzheimer's disease from healthy aging: evidence from functional MRI. Proc. Natl. Acad. Sci. U.S.A. 101, 4637-4642.

Gusnard, D. A., and Raichle, M. E. (2001). Searching for a baseline: functional imaging and the resting human brain. Nat. Rev. Neurosci. 2, 685-694.

Henson, R. (2005). A mini-review of fMRI studies of human medial temporal lobe activity associated with recognition memory. Q. J. Exp. Psychol. B 58, 340-360.

Herron, J. E. (2007). Decomposition of the ERP late posterior negativity: effects of retrieval and response fluency. Psychophysiology 44, 233-244.

Hoge, J., and Kesner, R. P. (2007). Role of CA3 and CA1 subregions of the dorsal hippocampus on temporal processing of objects. Neurobiol. Learn Mem. 88, 225-231.

Huijbers, W., Pennartz, C. M., Cabeza, R., and Daselaar, S. M. (2009). When learning and remembering compete: a functional MRI study. PLoS Biol. 7, el1. doi:10.1371/journal.pbio. 1000011

Huijbers, W., Pennartz, C. M., Cabeza, R., and Daselaar, S. M. (2011). The hippocampus is coupled with the default network during memory retrieval but not during memory encoding. PLoS ONE 6, e17463. doi:10.1371/journal.pone.0017463

Insausti, R., Juottonen, K., Soininen, H., Insausti, A. M., Partanen, K., Vainio, P., Laakso, M. P., and Pitkanen, A. (1998). MR volumetric analysis of the human entorhinal, perirhinal, and temporopolar cortices. AJNR Am. J. Neuroradiol. 19, 659-671.

Israel, S. L., Seibert, T. M., Black, M. L., and Brewer, J. B. (2010). Going their separate ways: dissociation of hippocampal and dorsolateral prefrontal activation during episodic retrieval and post-retrieval processing. J. Cogn. Neurosci. 22, 513-525.

Kawashima, R., O'sullivan, B. T., and Roland, P. E. (1995). Positronemission tomography studies of cross-modality inhibition in selective attentional tasks: closing the “mind's eye." Proc. Natl. Acad. Sci. U.S.A. 92, 5969-5972.

Kesner, R. P., Lee, I., and Gilbert, P. (2004). A behavioral assessment of hippocampal function based on a subregional analysis. Rev. Neurosci. 15, 333-351.

Kim, H. (2010). Dissociating the roles of the default-mode, dorsal, and ventral networks in episodic memory retrieval. Neuroimage 50, 1648-1657.

Kirwan, C. B., Jones, C. K., Miller, M. I., and Stark, C. E. (2007). Highresolution $\mathrm{AMRI}$ investigation of the medial temporal lobe. Hum. Brain Mapp. 28, 959-966.

Kirwan, C. B., and Stark, C. E. (2004). Medial temporal lobe activation during encoding and retrieval of novel face-name pairs. Hippocampus 14, 919-930.

Lacy, J. W., Yassa, M. A., Stark, S. M., Muftuler, L. T., and Stark, C. E. (2011). Distinct pattern separation related transfer functions in human CA3/dentate and CA1 revealed using high-resolution fMRI and variable mnemonic similarity. Learn. Mem. 18, 15-18.

Lee, I., and Kesner, R. P. (2004). Differential contributions of dorsal hippocampal subregions to memory acquisition and retrieval in contextual fear-conditioning. Hippocampus 14, 301-310.

Lepage, M., Habib, R., and Tulving, E. (1998). Hippocampal PET activations of memory encoding and retrieval: the HIPER model. Hippocampus 8, 313-322.

Leutgeb, J. K., Leutgeb, S., Moser, M. B., and Moser, E. I. (2007). Pattern separation in the dentate gyrus and CA3 of the hippocampus. Science 315, 961-966.

Maguire, E. A. (2001). Neuroimaging studies of autobiographical event memory. Philos. Trans. R. Soc. Lond. B Biol. Sci. 356, 1441-1451.

Mazoyer, B., Zago, L., Mellet, E., Bricogne, S., Etard, O., Houde, O., Crivello, F., Joliot, M., Petit, L., and Tzourio-Mazoyer, N. (2001). Cortical networks for working memory and executive functions sustain the conscious resting state in man. Brain Res. Bull. 54, 287-298.

McKiernan, K. A., Kaufman, J. N., Kucera-Thompson, J., and Binder, J. R. (2003). A parametric manipulation of factors affecting taskinduced deactivation in functional neuroimaging. J. Cogn. Neurosci. 15 394-408.

Meltzer, J. A., Negishi, M., and Constable, R. T. (2008). Biphasic hemodynamic responses influence deactivation and may mask activation in block-design fMRI paradigms. Hum. Brain Mapp. 29, 385-399.

Miller, M. I., Beg, M. F., Ceritoglu, C., and Stark, C. (2005). Increasing the power of functional maps of the medial temporal lobe by using large deformation diffeomorphic metric mapping. Proc. Natl. Acad. Sci. U.S.A. 102, 9685-9690.

Montaldi, D., Spencer, T. J., Roberts, N., and Mayes, A. R. (2006). The neural system that mediates familiarity memory. Hippocampus 16, 504-520.

Moscovitch, M. (1992). Memory and working-with-memory - a component process model based on modules and central systems. J. Cogn. Neurosci. 4, 257-267.

Nyberg, L., Habib, R., Mcintosh, A. R., and Tulving, E. (2000). Reactivation of encoding-related brain activity during memory retrieval. Proc. Natl. Acad. Sci. U.S.A. 97, 11120-11124.

Nyberg, L., Mcintosh, A. R., Houle, S., Nilsson, L. G., and Tulving, E. (1996). Activation of medial temporal structures during episodic memory retrieval. Nature 380, 715-717.

Raichle, M. E., Macleod, A. M., Snyder, A. Z., Powers, W. J., Gusnard, D. A., and Shulman, G. L. (2001). A default mode of brain function. Proc. Natl. Acad. Sci. U.S.A. 98, 676-682.
Ranganath, C., Cohen, M. X., Dam, C., and D'esposito, M. (2004a). Inferior temporal, prefrontal, and hippocampal contributions to visual working memory maintenance and associative memory retrieval. J. Neurosci. 24, 3917-3925.

Ranganath, C., Yonelinas, A. P., Cohen, M. X., Dy, C. J., Tom, S. M., and D'esposito, M. (2004b). Dissociable correlates of recollection and familiarity within the medial temporal lobes. Neuropsychologia 42, 2-13.

Rekkas, P. V., Westerveld, M., Skudlarski, P., Zumer, J., Pugh, K., Spencer, D. D., and Constable, R. T. (2005) Neural correlates of temporal-order judgments versus those of spatiallocation: deactivation of hippocampus may facilitate spatial performance. Brain Cogn. 59, 103-113.

Seibert, T. M., Gimbel, S. I., Hagler, D. J. Jr., and Brewer, J. B. (2011). Parietal activity in episodic retrieval measured by fMRI and MEG. $\mathrm{Neu}$ roimage 55, 788-793.

Shulman, G. L., Fiez, J. A., Corbetta, M., Buckner, R. L., Miezin, F. M., Raichle, M. E., and Petersen, S. E. (1997). Common blood flow changes across visual tasks.2. Decreases in cerebral cortex. J. Cogn. Neurosci. 9, 648-663. Smith, S. M., Jenkinson, M., Woolrich, M. W., Beckmann, C. F., Behrens, T. E., Johansen-Berg, H., Bannister, P. R., De Luca, M., Drobnjak, I., Flitney, D. E., Niazy, R. K., Saunders, J., Vickers, J., Zhang, Y., De Stefano, N., Brady, J. M., and Matthews, P. M. (2004). Advances in functional and structural MR image analysis and implementation as FSL. Neuroimage 23(Suppl. 1), S208-S219.

Sperling, R., Chua, E., Cocchiarella, A., Rand-Giovannetti, E., Poldrack, R., Schacter, D. L., and Albert, M. (2003). Putting names to faces: successful encoding of associative memories activates the anterior hippocampal formation. Neuroimage 20, 1400-1410.

Spreng, R. N., and Grady, C. L. (2010). Patterns of brain activity supporting autobiographical memory, prospection, and theory of mind, and their relationship to the default mode network. J. Cogn. Neurosci. 22, 1112-1123.

Squire, L. R., Wixted, J. T., and Clark, R. E. (2007). Recognition memory and the medial temporal lobe: a new perspective. Nat. Rev. Neurosci. 8, 872-883.

Stark, C. E., and Okado, Y. (2003). Making memories without trying: medial temporal lobe activity associated with incidental memory formation during recognition. J. Neurosci. 23, 6748-6753. 
Strange, B. A., Fletcher, P. C., Henson, R. N., Friston, K. J., and Dolan, R. J. (1999). Segregating the functions of human hippocampus. Proc. Natl. Acad. Sci. U.S.A. 96, 4034-4039.

Suthana, N., Ekstrom, A., Moshirvaziri, S., Knowlton, B., and Bookheimer, S. (2011). Dissociations within human hippocampal subregions during encoding and retrieval of spatial information. Hippocampus 21, 694-701.

Talairach, J., and Tornoux, P. (1988). Co-Planar Stereotaxic Atlas of the Human Brain: 3-Dimensional Proportional System: An Approach to Cerebral Imaging. Stuttgart: Georg Thieme.
Tulving, E., and Markowitsch, H. J. (1998). Episodic and declarative memory: role of the hippocampus. Hippocampus 8, 198-204.

Vincent, J. L., Kahn, I., Snyder, A. Z., Raichle, M. E., and Buckner, R. L. (2008). Evidence for a frontoparietal control system revealed by intrinsic functional connectivity. J. Neurophysiol. 100, 3328-3342.

Wais, P. E., Squire, L. R., and Wixted, J. T. (2010). In search of recollection and familiarity signals in the hippocampus. J. Cogn. Neurosci. 22, 109-123.

Woodruff, C. C., Johnson, J. D., Uncapher, M. R., and Rugg, M.
D. (2005). Content-specificity of the neural correlates of recollection. Neuropsychologia 43, 1022-1032.

Yonelinas, A. P., Otten, L. J., Shaw, K. N., and Rugg, M. D. (2005). Separating the brain regions involved in recollection and familiarity in recognition memory. J. Neurosci. 25, 3002-3008.

Conflict of Interest Statement: The authors declare that the research was conducted in the absence of any commercial or financial relationships that could be construed as a potential conflict of interest.
Received: 01 April 2011; accepted: 15 September 2011; published online: 30 September 2011.

Citation: Reas ET, Gimbel SI, Hales JB and Brewer JB (2011) Search-related suppression of hippocampus and default network activity during associative memory retrieval. Front. Hum. Neurosci. 5:112. doi: 10.3389/fnhum.2011.00112 Copyright (c) 2011 Reas, Gimbel, Hales and Brewer. This is an open-access article subject to a non-exclusive license between the authors and Frontiers Media $S A$, which permits use, distribution and reproduction in other forums, provided the original authors and source are credited and other Frontiers conditions are complied with. 